\title{
A Note On $G$-normal Distributions
}

\author{
Yongsheng Song*
}

January 16, 2015

\begin{abstract}
As is known, the convolution $\mu * \nu$ of two $G$-normal distributions $\mu, \nu$ with different intervals of variances may not be $G$-normal. We shows that $\mu * \nu$ is a $G$-normal distribution if and only if $\frac{\bar{\sigma}_{\mu}}{\underline{\sigma}_{\mu}}=\frac{\bar{\sigma}_{\nu}}{\underline{\sigma}_{\nu}}$.
\end{abstract}

Key words: $G$-normal distribution, Cramer's decomposition MSC-classification: $60 \mathrm{E} 10$

\section{Introduction}

Peng (2007) introduced the notion of $G$-normal distribution via the viscosity solutions of the $G$-heat equation below

$$
\begin{aligned}
\partial_{t} u-G\left(\partial_{x}^{2} u\right) & =0,(t, x) \in(0, \infty) \times \mathbb{R}, \\
u(0, x) & =\varphi(x),
\end{aligned}
$$

where $G(a)=\frac{1}{2}\left(\bar{\sigma}^{2} x^{+}-\underline{\sigma}^{2} x^{-}\right), a \in \mathbb{R}$ with $0 \leq \underline{\sigma} \leq \bar{\sigma}<\infty$, and $\varphi \in C_{b, \text { Lip }}(\mathbb{R})$, the collection of bounded Lipstchiz functions on $\mathbb{R}$.

Then the one-dimensional $G$-normal distribution is defined by

$$
N_{G}[\varphi]=u^{\varphi}(1,0)
$$

where $u^{\varphi}$ is the viscosity solution to the $G$-heat equation with the initial value $\varphi$. We denote by $\mathcal{G}^{0}$ the collection of functions $G$ defined above. For $G_{1}, G_{2} \in \mathcal{G}^{0}$, the convolution between $N_{G_{1}}, N_{G_{2}}$ is defined as $N_{G_{1}} * N_{G_{2}}[\varphi]:=N_{G_{1}}[\phi]$ with $\phi(x):=N_{G_{2}}[\varphi(x+\cdot)]$.

As is well-known, the convolution of two normal distributions is also normal. How about $G$-normal distributions? We state the question in the PDE language: For $G_{1}, G_{2} \in \mathcal{G}^{0}$, set $G(t, a)=G_{2}(a), t \in\left(0, \frac{1}{2}\right]$ and $G(t, a)=G_{1}(a), t \in\left(\frac{1}{2}, 1\right]$. Let $v^{\varphi}$ be the viscosity solution to the following PDE

$$
\begin{aligned}
\partial_{t} v-G\left(t, \partial_{x}^{2} v\right) & =0, \\
v(0, x) & =\varphi(x) .
\end{aligned}
$$

Can we find a function $G \in \mathcal{G}^{0}$ such that

$$
v^{\varphi}(1,0)=u^{\varphi}(1,0), \text { for all } \varphi \in C_{b, L i p}(\mathbb{R}) ?
$$

*Academy of Mathematics and Systems Science, CAS, Beijing, China, yssong@amss.ac.cn. Research supported by by NCMIS; Youth Grant of NSF (No. 11101406); Key Project of NSF (No. 11231005); Key Lab of Random Complex Structures and Data Science, CAS (No. 2008DP173182). 
Here $u^{\varphi}$ is the viscosity solution to the $G$-heat equation. However, [2] gave a counterexample to show that generally $N_{G_{1}} * N_{G_{2}}$ is not $G$-normal any more.

We denote by $\mathcal{G}$ the subset of $\mathcal{G}^{0}$ consisting of the elements with $\underline{\sigma}>0 . \quad N_{G}$ is called non-degenerate if $G$ belongs to $\mathcal{G}$. For $G \in \mathcal{G}$, set

$$
\beta_{G}:=\frac{\bar{\sigma}}{\underline{\sigma}} \text { and } \sigma_{G}=\frac{\bar{\sigma}+\underline{\sigma}}{2} .
$$

For abbreviation, we write $\beta, \sigma$ instead of $\beta_{G}, \sigma_{G}$ when no confusion can arise.

We shall prove that $N_{G_{1}} * N_{G_{2}}$ is a $G$-normal distribution if and only if $\beta_{G_{1}}=\beta_{G_{2}}$. Besides, the convolution between $G$-normal distributions is not commutative. We also prove that $N_{G_{1}} *$ $N_{G_{2}}=N_{G_{2}} * N_{G_{1}}$ if and only if $\beta_{G_{1}}=\beta_{G_{2}}$.

Therefore, in order to emphasize the importance of the ratio $\beta$, we denote the $G$-normal distribution $N_{G}$ by $N_{\beta}\left(0, \sigma^{2}\right)$.

\section{Characteristic Functions}

First we shall consider the solutions of special forms to the $G$-heat equation

$$
\partial_{t} u-G\left(\partial_{x}^{2} u\right)=0
$$

Assume that $u(t, x)=a(t) \phi(x)$ with $a(t) \geq 0$ is a solution to the $G$-heat equation (2.1). Then we conclude that

$$
\frac{a^{\prime}(t)}{a(t)}=\frac{G\left(\phi^{\prime \prime}(x)\right)}{\phi(x)}
$$

is a constant. Assuming that they are equal to $-\frac{\rho^{2}}{2}$, we have $a(t)=e^{-\frac{\rho^{2}}{2} t}$ and

$$
G\left(\phi^{\prime \prime}(x)\right)=-\frac{\rho^{2}}{2} \phi(x)
$$

If $\phi^{\prime \prime}(x)$ is positive, the equation $(2.2)$ reduces to

$$
\bar{\phi}^{\prime \prime}(x)=-\frac{\rho^{2}}{\bar{\sigma}^{2}} \bar{\phi}(x) .
$$

and the solution is

$$
\bar{\phi}(x)=\bar{\lambda} \cos \left(\frac{\rho}{\bar{\sigma}} x+\bar{\theta}\right) .
$$

On the other hand, if $\phi^{\prime \prime}(x)$ is negative, the equation (2.2) reduces to

$$
\underline{\phi}^{\prime \prime}(x)=-\frac{\rho^{2}}{\underline{\sigma}^{2}} \underline{\phi}(x) .
$$

and the solution is

$$
\underline{\phi}(x)=\underline{\lambda} \cos \left(\frac{\rho}{\sigma} x+\underline{\theta}\right) .
$$

Motivated by the arguments above, we shall construct the solutions to the equation (2.2) in the following way. Denoting by $\beta=\frac{\bar{\sigma}}{\underline{\sigma}}$, set

$$
\phi_{\beta}(x)= \begin{cases}\frac{2}{1+\beta} \cos \left(\frac{1+\beta}{2} x\right) & \text { for } x \in\left[-\frac{\pi}{1+\beta}, \frac{\pi}{1+\beta}\right) \\ \frac{2 \beta}{1+\beta} \cos \left(\frac{1+\beta}{2 \beta} x+\frac{\beta-1}{2 \beta} \pi\right) & \text { for } x \in\left[\frac{\pi}{1+\beta}, \frac{(2 \beta+1) \pi}{1+\beta}\right) .\end{cases}
$$




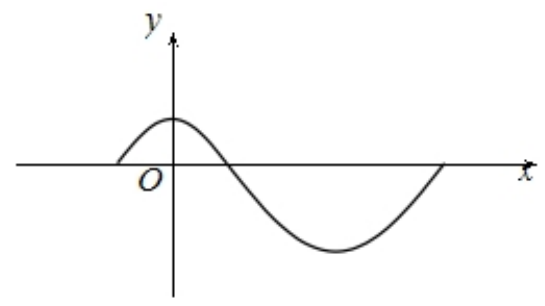

Figure 1: $\phi_{\beta}(x)$

This is a variant of the trigonometric function $\cos x$ (see Figure 1).

Then extend the definition of $\phi_{\beta}$ to the whole real line by the property $\phi(x+2 k \pi)=\phi(x)$, $k \in \mathbb{Z}$. Clearly, $\phi_{1}(x)=\cos x$ and $\phi_{\beta}$ belongs to $C^{2,1}(\mathbb{R})$, the space of bounded functions on $\mathbb{R}$ with uniformly Lipschitz continuous second-order derivatives.

Proposition $2.1 \phi_{\beta}$ is a solution to equation (2.2) with $\rho=\frac{\underline{\sigma}+\bar{\sigma}}{2}=: \sigma$.

Proof. The proof follows from simple calculations. For $x \in\left[-\frac{\pi}{1+\beta}, \frac{\pi}{1+\beta}\right)$,

$$
\phi_{\beta}^{\prime \prime}(x)=-\frac{1+\beta}{2} \cos \left(\frac{1+\beta}{2} x\right)=-\frac{(1+\beta)^{2}}{4} \phi_{\beta}(x) \leq 0 .
$$

So

$$
G\left(\phi_{\beta}^{\prime \prime}(x)\right)=-\frac{(1+\beta)^{2} \underline{\sigma}^{2}}{8} \phi_{\beta}(x)=-\frac{\sigma^{2}}{2} \phi_{\beta}(x)
$$

For $x \in\left[\frac{\pi}{1+\beta}, \frac{(2 \beta+1) \pi}{1+\beta}\right)$,

$$
\phi_{\beta}^{\prime \prime}(x)=-\frac{1+\beta}{2 \beta} \cos \left(\frac{1+\beta}{2 \beta} x+\frac{\beta-1}{2 \beta} \pi\right)=-\frac{(1+\beta)^{2}}{4 \beta^{2}} \phi_{\beta}(x) \geq 0 .
$$

So

$$
G\left(\phi_{\beta}^{\prime \prime}(x)\right)=-\frac{(1+\beta)^{2} \bar{\sigma}^{2}}{8 \beta^{2}} \phi_{\beta}(x)=-\frac{\sigma^{2}}{2} \phi_{\beta}(x)
$$

Corollary 2.2 Let $\sigma:=\frac{\underline{\sigma}+\bar{\sigma}}{2}$ and $\beta:=\frac{\bar{\sigma}}{\sigma} \cdot e^{-\frac{\sigma^{2}}{2} t} \phi_{\beta}(x)$ is the solution to equation (2.1) with the initial value $\phi_{\beta}(x)$. In other words, $N_{G}\left[\phi_{\beta}(x+\sqrt{t} \cdot)\right]=e^{-\frac{\sigma^{2}}{2} t} \phi_{\beta}(x)$.

For $\lambda>0$ and $c, \theta \in R$, set $\phi_{\beta}^{\lambda, c, \theta}(x):=\lambda \phi_{\beta}(c x+\theta)$. It's easy to check that

$$
G\left(\left(\phi_{\beta}^{\lambda, c, \theta}\right)^{\prime \prime}\right)=-\frac{c^{2} \sigma^{2}}{2} \phi_{\beta}^{\lambda, c, \theta}
$$

So $e^{-\frac{c^{2} \sigma^{2}}{2} t} \phi_{\beta}^{\lambda, c, \theta}(x)$ is the solution to equation (2.1) with the initial value $\phi_{\beta}^{\lambda, c, \theta}(x)$. For any $\beta>1$, we call

$$
\left(\phi_{\beta}^{\lambda, c, \theta}(x)\right)_{\lambda, c, \theta}
$$

the characteristic functions of $G$-normal distributions $N_{\beta}\left(0, \sigma^{2}\right)$. 


\section{Cramer's Type Decomposition for G-normal Distributions}

Let's introduce more properties on the characteristic functions $\left\{\phi_{\beta}(x)\right\}_{\beta \geq 1}$. For $1 \leq \alpha<\beta<\infty$, we have

$$
\phi_{\alpha}(x)-\phi_{\beta}(x) \geq \frac{2(\beta-\alpha)}{(1+\alpha)(1+\beta)}=: e(\alpha, \beta)>0 .
$$

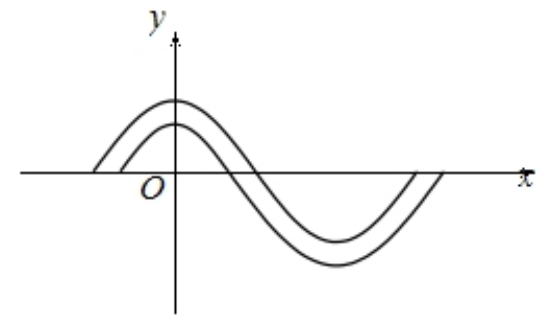

Figure 2: $\phi_{\alpha}(x)$ and $\phi_{\beta}(x)$

Theorem 3.1 For $G_{1}, G_{2} \in \mathcal{G}$, the convolution $N_{G_{1}} * N_{G_{2}}$ is a G-normal distribution if and only if $\beta_{G_{1}}:=\frac{\bar{\sigma}_{G_{1}}}{\underline{\sigma}_{G_{1}}}=\frac{\bar{\sigma}_{G_{2}}}{\underline{\sigma}_{G_{2}}}=: \beta_{G_{2}}$.

Proof. The sufficiency is obvious, and we only prove the necessity. Assume $N:=N_{G_{1}} * N_{G_{2}}$ is a $G$-normal distribution. Then $\underline{\sigma}_{N}^{2}=\underline{\sigma}_{G_{1}}^{2}+\underline{\sigma}_{G_{2}}^{2}$ and $\bar{\sigma}_{N}^{2}=\bar{\sigma}_{G_{1}}^{2}+\bar{\sigma}_{G_{2}}^{2}$. If $\beta_{G_{1}} \neq \beta_{G_{2}}$, we may assume that $\beta_{G_{1}}<\beta_{G_{2}}$, and the other case can be proved similarly. Then we have $\beta_{G_{1}}<\beta_{N}<\beta_{G_{2}}$. On one hand we have

$$
N\left[\phi_{\beta_{N}}(\sqrt{t} \cdot)\right]=e^{-\frac{\sigma_{N}^{2}}{2} t} \phi_{\beta_{N}}(0)=\frac{2}{1+\beta_{N}} e^{-\frac{\sigma_{N}^{2}}{2} t} .
$$

On the other hand we have

$$
\begin{aligned}
& N_{G_{2}}\left[\phi_{\beta_{N}}(\sqrt{t}(x+\cdot))\right] \\
\geq & N_{G_{2}}\left[\phi_{\beta_{G_{2}}}(\sqrt{t}(x+\cdot))\right]+e\left(\beta_{N}, \beta_{G_{2}}\right) \\
= & \left.e^{-\frac{\sigma_{G_{2}}^{2}}{2} t} \phi_{\beta_{G_{2}}}(\sqrt{t} x)\right]+e\left(\beta_{N}, \beta_{G_{2}}\right) \\
\geq & -\frac{2 \beta_{G_{2}}}{1+\beta_{G_{2}}} e^{-\frac{\sigma_{G_{2}}^{2}}{2} t}+e\left(\beta_{N}, \beta_{G_{2}}\right) .
\end{aligned}
$$

Consequently,

$$
N\left[\phi_{\beta_{N}}(\sqrt{t} \cdot)\right]=N_{G_{1}} * N_{G_{2}}\left[\phi_{\beta_{N}}(\sqrt{t} \cdot)\right] \geq-\frac{2 \beta_{G_{2}}}{1+\beta_{G_{2}}} e^{-\frac{\sigma_{G_{2}}^{2}}{2} t}+e\left(\beta_{N}, \beta_{G_{2}}\right) .
$$

Combining the above arguments we have

$$
\frac{2}{1+\beta_{N}} e^{-\frac{\sigma_{N}^{2}}{2} t} \geq-\frac{2 \beta_{G_{2}}}{1+\beta_{G_{2}}} e^{-\frac{\sigma_{G_{2}}^{2}}{2} t}+e\left(\beta_{N}, \beta_{G_{2}}\right) \text {, for any } t>0,
$$

which is a contradiction noting that $e\left(\beta_{N}, \beta_{G_{2}}\right)>0$. 
Theorem 3.2 For $G_{1}, G_{2} \in \mathcal{G}, N_{G_{1}} * N_{G_{2}}=N_{G_{2}} * N_{G_{1}}$ if and only if $\beta_{G_{1}}=\beta_{G_{2}}$.

Proof. We shall only prove the necessity. Assume that $\beta_{G_{1}}<\beta_{G_{2}}$. Then

$$
N_{G_{1}}\left[\phi_{\beta_{G_{1}}}(\sqrt{t} x+\sqrt{t} \cdot)\right]=e^{-\frac{\sigma_{G_{1}}^{2}}{2} t} \phi_{\beta_{G_{1}}}(\sqrt{t} x) \leq \frac{2}{1+\beta_{G_{1}}} e^{-\frac{\sigma_{G_{1}}^{2}}{2} t} .
$$

So

$$
N_{G_{2}} * N_{G_{1}}\left[\phi_{\beta_{G_{1}}}(\sqrt{t} \cdot)\right] \leq \frac{2}{1+\beta_{G_{1}}} e^{-\frac{\sigma_{G_{1}}^{2}}{2} t}
$$

On the other hand

$$
\begin{aligned}
& N_{G_{2}}\left[\phi_{\beta_{G_{1}}}(\sqrt{t} x+\sqrt{t} \cdot)\right] \\
\geq & N_{G_{2}}\left[\phi_{\beta_{G_{2}}}(\sqrt{t} x+\sqrt{t} \cdot)\right]+e\left(\beta_{G_{1}}, \beta_{G_{2}}\right) \\
= & e^{-\frac{\sigma_{G_{2}}^{2}}{2} t} \phi_{\beta_{G_{2}}}(\sqrt{t} x)+e\left(\beta_{G_{1}}, \beta_{G_{2}}\right) \\
\geq & -\frac{2 \beta_{G_{2}}}{1+\beta_{G_{2}}} e^{-\frac{\sigma_{G_{2}}^{2}}{2} t}+e\left(\beta_{G_{1}}, \beta_{G_{2}}\right),
\end{aligned}
$$

which implies that

$$
N_{G_{1}} * N_{G_{2}}\left[\phi_{\beta_{G_{1}}}(\sqrt{t} \cdot)\right] \geq-\frac{2 \beta_{G_{2}}}{1+\beta_{G_{2}}} e^{-\frac{\sigma_{G_{2}}^{2}}{2} t}+e\left(\beta_{G_{1}}, \beta_{G_{2}}\right) .
$$

Noting that $e\left(\beta_{G_{1}}, \beta_{G_{2}}\right)>0$, we have

$$
N_{G_{1}} * N_{G_{2}}\left[\phi_{\beta_{G_{1}}}(\sqrt{t} \cdot)\right]>N_{G_{2}} * N_{G_{1}}\left[\phi_{\beta_{G_{1}}}(\sqrt{t} \cdot)\right] \text { for } t \text { large enough. }
$$

\section{References}

[1] Peng, S.(2007) G-expectation, G-Brownian Motion and Related Stochastic Calculus of Itô type, Stochastic analysis and applications, 541-567, Abel Symp., 2, Springer, Berlin.

[2] Hu, M. (2010) Nonlinear Expectations and Related Topics, Doctoral thesis. 